\title{
The First Observation of 3-Dimensional Motion and Twist in Sperm Flagella of the Stag Beetle Prosopocoilus inclinates
}

\author{
Masaru IRIE, Miyoko KUBO-IRIE ${ }^{1)}$ and Hideo MOHRI ${ }^{2)}$ \\ Waseda University, Tokyo 169-8555, Japan \\ 1) University of Air, Chiba 261-8586, Japan \\ 2) National Institute for Basic Biology, Okazaki 444-8585, Japan \\ \& University of Tokyo, Komaba 113-0033, Japan
}

(Received 6 December 2006 / Accepted 18 April 2007)

\begin{abstract}
We discovered the 3-dimensional twist motion of sperm flagella of the stag beetle Prosopocoilus inclinates. The morphological features are discussed with experimental data obtained through various 'imaging techniques' including those developed in thermo-nuclear fusion research. The helical deformation length observed in the optical micrograph agreed statistically with those of transmission electron micrographs (TEM) on both ultra-thin section and negatively stained samples. This indicated that the helical twist mechanism of flagellar axoneme could be safely discussed from TEM. In order to elucidate this, we applied the newly developed Constrained Electron Beam Tomography (CEBT) technique adapted from our unique fusion plasma diagnosis. This requires basic assumptions of "the optimum deformation" and "the coherent length" as mathematical constraints. The results are the key parameters of the flagellum deformation, e.g. the helical pitch (HP) of both axoneme and mitochondrial derivatives as well as the phase slip (PS) between them. They allow the quantitative discussion on this motion.
\end{abstract}

(C) 2007 The Japan Society of Plasma Science and Nuclear Fusion Research

Keywords: Insect sperm flagella, 3-dimensional helical motion, Constrained Electron Beam Tomography (CEBT), coherent length (CL), helical pitch (HP), phase slip (PS)

DOI: $10.1585 /$ pfr.2.S1028

\section{Inroduction}

The insect spermatozoon is filiform with a long flagellum consisted of two mitochondrial derivatives and the $9+9+2$ microtubules [1]. In addition to these ordinary components, the sperm flagellum of the stag beetles had a centriole adjunct characterized by a unique structure [2]. The relationship between this flagellar configuration and the motion is not well understood.

The early study of insect sperm motility was examined under a dark field microscope and recorded by cinematographic methods [3]. According to Woolley (1977), motile spermatozoa of the golden hamster have been arrested by rapid freezing and then fixed with glutaraldehyde. The flagellar waveforms stabilized were similar to those in living sperm [4]. In this paper, we analyzed the motion pattern of stag beetle sperm assuming that the flagellar movement of living sperm was similar to the fixed motile pattern. Furthermore, the soft X-ray tomography technique in plasma fusion research was modified into CEBT (the Constrained Electron Beam Tomography) [5] and applied for negatively stained sperm samples. This allows image reconstruction from smaller peripheral scanning data by setting basic assumptions as constraints.

\section{Materials and Methods 2.1 Insects}

Adult males of the stag beetle Prosopocoilus inclinates were supplied from an insect farm. A pair of follicular testes and convoluted seminal vesicle in the dorsal vessel were dissected out in the modified Kiev solution and used as samples. Individual spermatozoa obtained from the seminal vesicle were freed into the solution.

\subsection{Electron micrograph and specimen preparation}

Spermatozoa were directly placed into primary fixative $(2.5 \%$ glutaraldehyde and $1 \%$ tannic acid in $0.2 \mathrm{M}$ sodium cacodylate buffer at $\mathrm{pH}$ 7.4). After post-fixation with $1 \%$ OsO4, samples were dehydrated through graded levels of ethanol and embedded in Quetol 812 (Nissin EM). The ultrathin-sections were made with a Sorvall ultramicrotome (Leica), stained doubly with uranylacetate and lead citrate, and then observed using a JEOL 1200 EX II electron microscope. For observation of whole spermatozoa, drops of prefixed samples were deposited onto carbonfilm-coated copper grids that had been rinsed with deionized water. After being negatively stained with a drop of $1 \%$ uranyl acetate, the grids were drained, air-dried and examined with the electron microscope. 


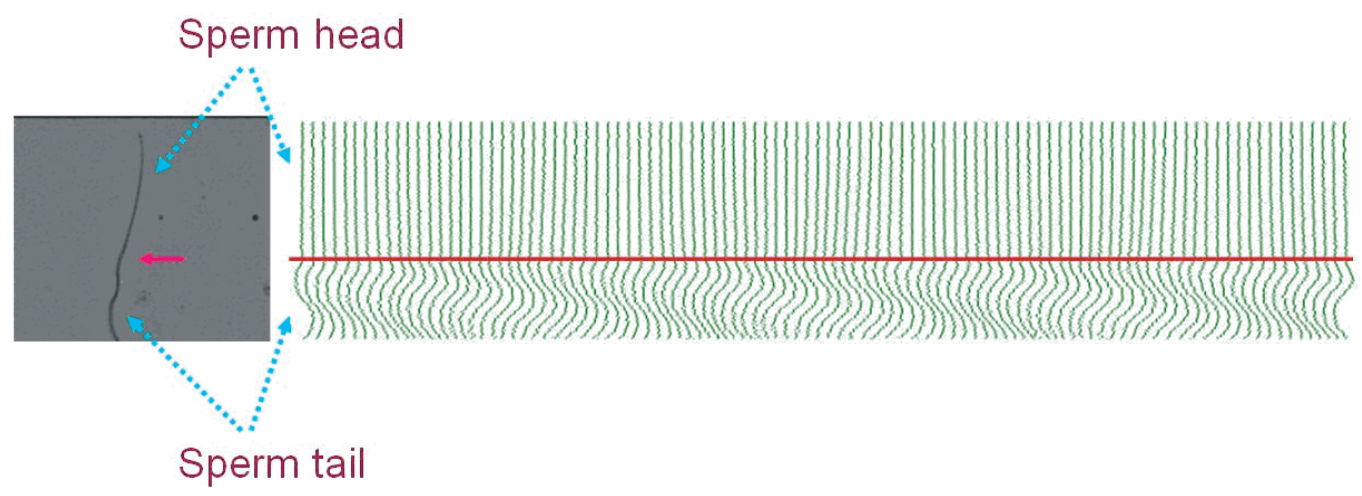

Fig. 1 Optical micrograph and the extracted time history of the stag beetle sperm movements. (time separation 2 ms) The flagellar motion initiates at the posterior end of the nucleus (arrow) and is transferred to the sperm tail.

\subsection{Constrained Electron Beam Tomogra- phy (CEBT): flagellar observation}

This fundamental and well established tomography technique requires fine and consecutive peripheral $180 \mathrm{de}-$ gree observation of the specimen. In the flagellar observation, we met the famous "observation angle problem". In order to irradiate the electron beam to the specimen from different angles, we should tilt the specimen, but the electromagnetic lens configuration limits the selectable angles.

One of the authors had met the same problems in thermo-nuclear fusion experiments in the Spherical Tokamak device, FBX-II, in Waseda University and in the Reverse Field Pinch device, TPE-2M, in Electro Technical Laboratory. Because of the number of the observation ports, the detection angles are limited in these devices. He developed Constrained Soft X-Ray Tomography method (CSXT) assuming the coherent length (CL) and semi-rigid rotator model. The obtained results are not the detailed plasma profile but only the transition between lower Fourier mode of the Magneto Hydro-Dynamic features of the fusion plasma [5].

In flagellar diagnostics, the deformation of the cross sectional structure should be anticipated. We defined the zone length "the coherent length" within which the inner structure is virtually identical or the self similarity is maintained. This condition can be summarized as below.

1. In $\mathrm{CL}$, the deformation of axoneme $(\mathrm{AX})$ and annex (AN) is negligible.

2. In CL, the twist pitch of $A X$ and $A N$ can be different but constant. (Phase Slip: PS).

If $\mathrm{CL}$ is long enough, the observation angle effectively covers full one twist cycle. Then the conventional tomography technique becomes applicable. We name this condition as the coherency condition (CC) and this technique as the Constrained Electron Beam Tomography (CEBT).

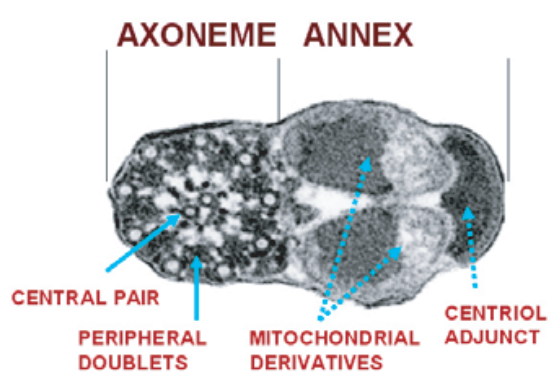

Fig. 2 Cross section of the sperm flagellum.

\section{Results}

\subsection{Observation of the living sperm move- ment}

The motion of sperm from the seminal vesicle was monitored under the phase contrast setup of the optical microscope Olympus IX71 and recorded with NAC HiD CAM high speed camera with the capture speed of up to 5000 frame/second. With this function we could eliminate the aliasing problems. In Fig. 1 the computer reconstructed data showing the deviation from the first frame is displayed. The time separations between each consecutive frame are $2 \mathrm{~ms}$. The flagellar motion was originated in the zone that linked the sperm head with the sperm tail (red arrow) and three-dimensional helical flagellar motion was transmitted to the bottom. The sperm head is about 40 micro-meter.

\subsection{Electron micrograph of flagellar cross section}

The main element of flagella and cilia responsible for their motion is the axoneme (AX) with 9 peripheral doublet microtubules and a central pair of microtubules. The 2-dimensional motion is activated by peripheral doublets with dynein arms (6). The sperm flagellum of the stag beetle has more complicated structure (Fig. 2). Just as the other insects, it has 9 additional peripheral singlet microtubules 
which makes $9+9+2$ configuration. It also have an annex (AN) with two mitochondrial derivatives (MD) partly covered by the centriole adjunct (CA).

\subsection{Electron micrograph of the flagellar lon- gitudinal section}

To find out the inner structure, we have prepared "the ultra-thin sectioned specimen" and observed the longitudinal sections under the electron microscope. In Fig. 3, the torsion of the flagellar components was observed; i.e. the parallel arrangements of $\mathrm{AX}, \mathrm{MD}$ and CA are twisted and reversed twice. The electron dense portion is MD. The CA was not on the sectioned plane. With this method, however, we could observe AX structure from only one angle in one specimen.

In Fig. 4, we show the simple model of the extracted flagellar components which seemed to affect the motion. The large helical structure (LHS) is the complex composed of AX and MD. The compact helical structure (CHS) is the AX itself. From Fig. 2 and Fig. 3, we found the helical twist in both LHS and CHS. We are interested in the pitches (HP: helical pitch) of these twist and the phase dif-

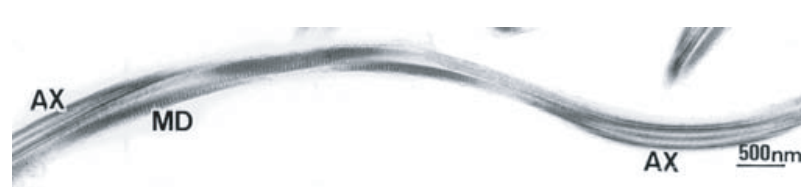

Fig. 3 Ultra-thin section to identify the inner structure.

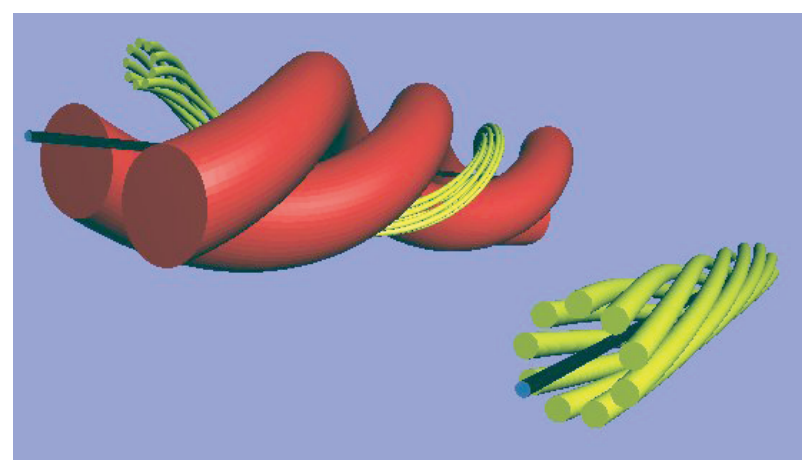

Fig. 4 Helical twist AX (green), MD(red) and the imaginary twist axis(black) Left: large helical structure (LHS): AX and MD Right: compact helical structure (CHS) : AX itself. ference (PS: phase slip). If the configuration satisfies the coherency condition (CC), we can identify the HP and PS safely from the TEM. This allows the quantitative discussion of the flagellar helical motion.

\subsection{Electron micrograph of the negatively stained flagellum}

In order to obtain this information, we applied our CEBT technique. The whole spermatozoon was stained negatively; the sperm tail was undulated to be seen as the transmitted image. In Fig. 5, we displayed the TEM image of the twisted flagellum. The MD are observed as a dark portion just as the ultra thin section in Fig. 3. However, in this case, the information of AX was inherent in this micrograph. The flagellum was seen to be in sinusoidal and well within the statistical average of the live flagellar wave length. From this micrograph, we can see the strong correlation between the bending angle of the flagellum and the observed width.

\subsection{Transmission electron beam tomogra- phy}

In Fig. 6, we display the results from the conventional tomography simulation. On the left end we placed the image of the sperm flagellum shown in Fig. 2. We rotate this image by 180 degree under the collimated electron beam. The numerically integrated image corresponding to the transmitted image against the rotational angle is on the right. The standard "Back Projection" leads the blur image on the further right figure and the blur image is deconvoluted to obtain the rightmost picture.

\subsection{Helical pitch (HP) and phase slip (PS) measurement}

If the coherency condition (CC) is violated, the tomographic image would be smeared out locally by the 'noise'.

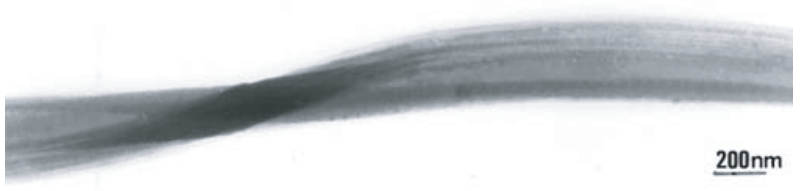

Fig. 5 Local TEM image of the twisted flagellum, negatively stained: Raw data for electron beam tomography.

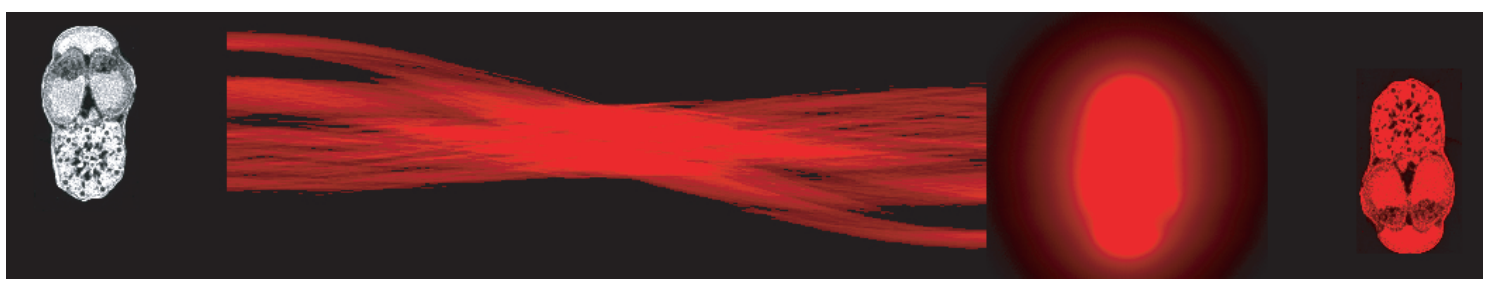

Fig. 6 Conventional tomography. 

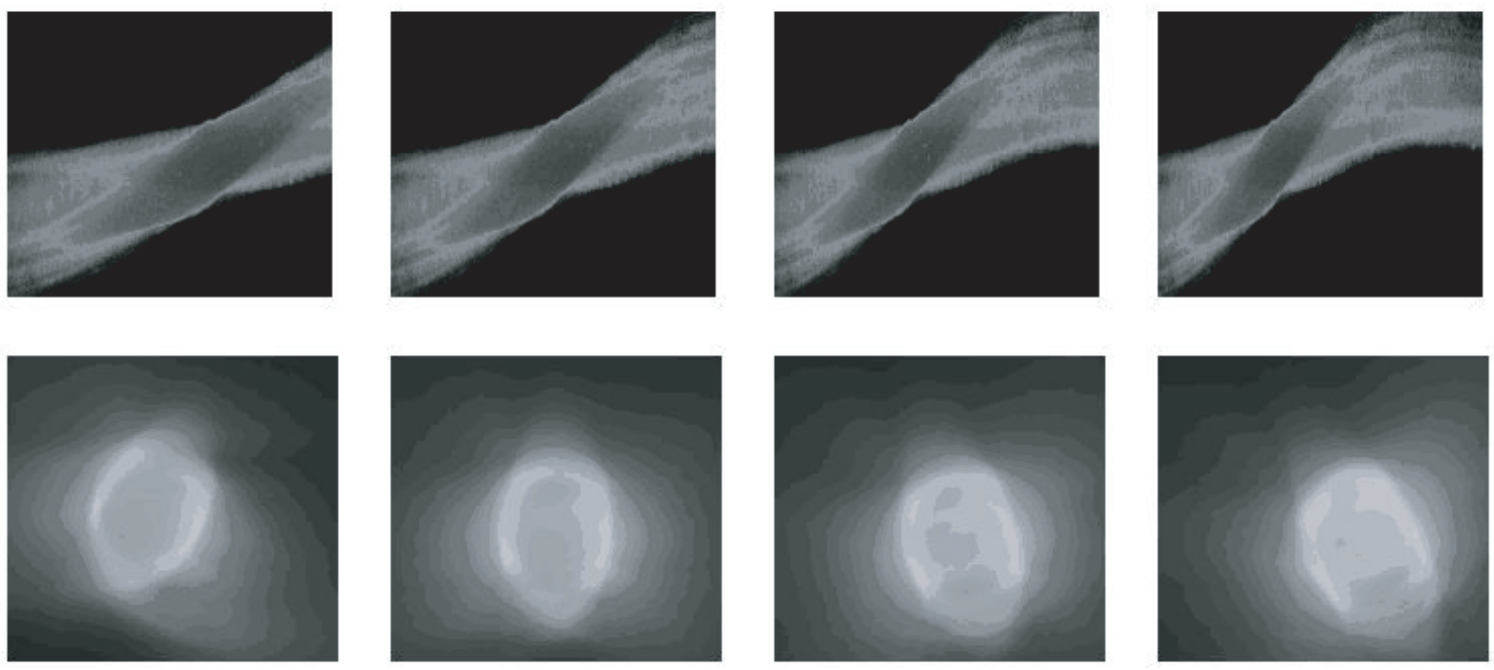

Fig. 7 Example of the helical pitch (HP) and phase slip (PS) measurement Top. Source data from Fig. 5. Bottom: Tomographic blur picture to identify CL.

In other words, we can detect CL, helical pitch and phase slip. In the final figure, Fig. 7, we present examples of the measurement. The top pictures are the extracted and fitted images to one cycle from the twisted flagellum taken from Fig. 5. The extracted horizontal pixel lengths are 3000, 4000, 5000 and 6000 consecutively from left to right. The bottom pictures are the tomographic blur pictures obtained from the corresponding image above. We can see the effect of CL, HP and PS from those pictures. The detailed discussion on this result is to be published elsewhere.
[1] B.G.M. Jamieson, R. Dallai and B.A. Afzelius, Order Hemiptera. In: INSECTS. Their spermatozoa and phylogeny (Science Publishers UK, 1999) p.277.

[2] M. Kubo-Irie, I. Miura, M. Irie , T. Nakazawa and H. Mohri, Invertebrate Reproduction and Development 37, 223 (2000).

[3] B. Baccetti, A.G. Burrini, R. Dallai, F. Giusti, M. Mazzini, T. Renieri, F. Rosati and G. Selmi, Journal of Mechanochemical Cell Motility 2,149 (1973).

[4] D.M. Woolley, The Journal of Cell Biology 75, 851 (1977).

[5] T. Umemoto, K. Hattori, Y. Sato, K. Hayase and M. Irie, Journal Plasma and Fusion Research. 72, 1082 (1996).

[6] C.J. Brokaw, Science 243, 1593 (1989). 